\title{
MONITOREO APLICADO A LA ELABORACIÓN DE UN PLAN DE CONSERVACIÓN DE Calycophyllum candidissimum EN FRAGMENTOS DE BOSQUE SECO DEL SUROESTE DE NICARAGUA
}

\author{
MONITORING APPLIED TO THE PREPARATION OF A CONSERVATION PLAN OF \\ Calycophyllum candidissimum IN FRAGMENTS OF A DRYFOREST IN THESOUTHWEST \\ OF NICARAGUA
}

\author{
Noguera-Talavera Álvaro, González-Rivas Benigno, Castro-Marín Guillermo \\ Facultad de Recursos Naturales y del Ambiente \\ Alvaro.Noguera@una.edu.ni,Benigno.Gonzalez@una.edu.ni, Guillermo.Castro@una.edu.ni
}

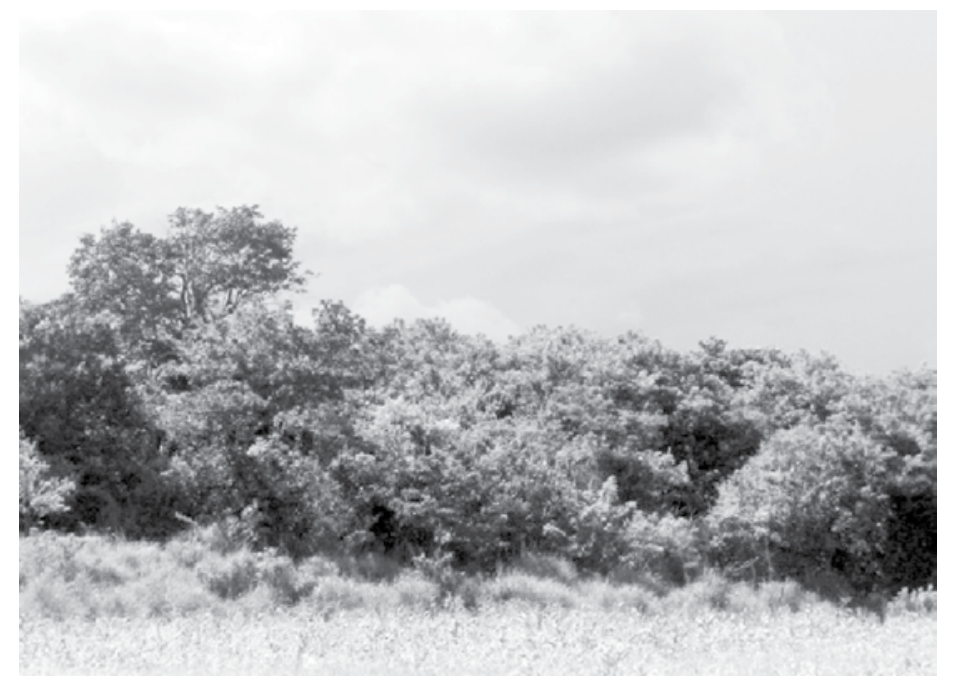

\section{RESUMEN}

La fragmentación de bosques por actividades antropogenicas ha generado cambios drásticos en las características poblacionales de algunas especies, hasta el punto de presentar altos niveles de amenaza en ciertas localidades. A partir de la elaboración y análisis de una línea base de monitoreo se propone la implementación en la zona Sur de un plan de conservación de la especie Calycophyllum candidissimum. La línea base está constituida por información de parámetros poblacionales de la especie; elementos que forman parte de tres criterios y 12 indicadores definidos para la ejecución de un monitoreo efectivo y a largo plazo que enriquezca los elementos del plan de conservación. Los resultados del monitoreo sugieren baja densidad de la especie en fragmentos de 0.5 a 10 hectáreas. La relación entre mortalidad y reclutamiento muestra pérdida progresiva de los individuos en las poblaciones monitoreadas; aun cuando datos de regeneración natural indican un cambio positivo en la densidad en un periodo de tres años. La propuesta de plan
Fragmentationof forests by anthropogenic activities has led to drastic changes in the population characteristics of some species causing thre at levels in certain localities. From the design and analysis of a monitoring base line, it is proposed the implementation of a conservation plan of the specie Calycophyllum candidissimumin two fragment of the southpacific of Nicaragua. Base line informationconsistsof population parameters of the specie, elements that are part of three criteriaand 12 indicators defined forthe implementation of aneffective long-termmonitoringthat enriches the elements of the conservation plan. Sampling results show low density of the species in small fragments (from 0.5 to $10 \mathrm{ha}$ ). The relationship between mortality and recruitment shows progressive loss of individuals in populations monitored, even if natural regeneration data indicate a positive change in density over a period of three years. The proposed conservation plan consists of four strategies that reflect the need to complete the monitoring, 
de conservación está constituida por cuatro estrategias que reflejan la necesidad de completar monitoreo, la protección del hábitat, y el restablecimiento de la población de Calycophyllum candidissimum; además de un modelo que define buenas prácticas de manejo y conservación de la especie; y establece actividades puntuales para operativizar la estrategia. Palabras clave: Fragmentación, disminución de la población, plan de conservación. habitat protection and restoration of Calycophyllum candidissimum population, in addition to a model that defines good management practices and conservation of the species; and sets out specific activities to operationalize the strategy. Keywords: Fragmentation, population decrease,conservation plan.

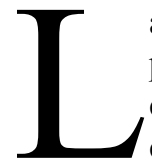
a región del Pacífico de Nicaragua es dominada por vegetacion típica de bosque tropical seco, el cual se encuentra altamente fragmentado, como consecuencia de que un $60 \%$ de la población del país está asentada en áreas cercanas al bosque. El monitoreo se concibe como la observación y recopilación continua de eventos típicos del bosque y a través de parámetros permite caracterizar y diagnosticar elementos de interés. Comúnmente, el monitoreo con fines prácticos de aplicación debe contestar a ciertas preguntas básicas como: ¿Por qué monitorear?, ¿Para qué monitorear? y ¿Qué monitorear? La necesidad de monitoreo de la especie C. candidissimumse fundamenta en la disminución de la abundancia de la especie, causada por uso excesivo, y la fragmentación del bosque. El fenómeno descrito hace que $\mathrm{C}$. candidissimum sea una especie con alto potencial de amenaza local en fragmentos de bosque seco secundario del Pacifico.

En cuanto a ¿para qué monitorear? se establece como propósito el documentar los factores (ambientales y socioeconómicos) que afectan la dinámica poblacional de $\mathrm{C}$. candidissimum, lo cual permitirá la toma de decisiones en relación a los componentes de un plan de manejo adecuado a las condiciones naturales de la especie y condiciones del sitio.

En relación a qué monitorear, se definió como elemento clave de prioridad la conservación de la especie C. candidissimum; sin embargo, para la conservación de cualquier especie, es determinante la complejidad de relaciones con el hábitat, por lo que la conservación de una especie debe partir del monitoreo de su hábitat.

Este artículo presenta resultados de un sistema de monitoreo llevado a cabo por más de cinco años, y queha permitido registrar información de los cambios en la estructura de dos fragmentos del bosque seco del Suroeste de Nicaragua; y cuya aplicación aportó elementos sustanciales para la elaboración de una propuesta de plan de conservación de la especie C. candidissimum.

\section{MATERIALES Y MÉTODOS}

Ubicación del área propuesta para ejecutar el plan de conservación. El área seleccionada para implementar el plan de conservación se ubica en la localidad Río Medina; ubicada aproximadamente a $10 \mathrm{Km}$ al Suroeste de la ciudad de Nandaime en el departamento de Granada.
Características de las áreas propuestas para implementar el plan de conservación. En el área se presentan promedios anuales deprecipitación de $1444 \mathrm{~mm}$ y una temperatura de $27^{\circ} \mathrm{C}$. La vegetación en el área es clasificada como una formación de bosque tropical seco deciduo (Ponce y Montalbán, 2005). La altitud varía entre 92 y 167 metros sobre el nivel del mar.

La Chipopa. Presenta un estado sucesional en edad de 18 años, con una vegetación ya establecida (Castro et al., 2005). Cuenta con área de tacotal de 3.83 hectáreas, localizada entre las coordenadas 11\%42'31" Norte y $86^{\circ} 05^{\prime} 08^{\prime}$ 'Oeste. Siendo su altitud promedio de $151 \mathrm{msnm}$.

La Zorra. Presenta un estado sucesional en edad de 13 años, con una vegetación con elementos arbustivos y especies arbóreas en fase de establecimiento (Castro et al., 2005). El área del bosque tiene una extensión de 4.21 hectáreas y se encuentra localizado entre los $11^{\circ} 41^{\prime} 42^{\prime \prime}$ Norte y 86 04 '25" Oeste, a una altitud de 131 msnm (Ponce y Montalbán, 2005).

Levantamiento de la información. La información que aportó elementos a la elaboración de una propuesta de plan de conservación, se recopiló a través de la construcción de una línea base constituida por diversos estudios que reportan valores de abundancia de la especie en fragmentos de bosque en algunos sitios de Nicaragua, referencias sobre los trabajos en mención son las siguientes:

Sánchez et al., 2004 realizaron una caracterización para conocer la diversidad, composición y estructura de la vegetación en un agropaisaje ganadero de Matiguás.

Sánchez et al., 2005 realizaron una caracterización para conocer la diversidad, composición y estructura de la vegetación en un agropaisaje de la zona Sur de Nicaragua. González-Rivas, 2005. Estudio la diversidad de especies en áreas de bosque seco de la zona Sur. Noguera et al., 2009. Determinó la diversidad, composición y dinámica poblacional de dos fragmentos de bosque seco con diferentes estados sucesionales (Tabla 3).

Levantamiento de información en el campo. La información sobre aspectos de dinámica poblacional de $\mathrm{C}$. candidissimum fue obtenida a través del registro periódico de información estructural en parcelas de muestreo permanentes 
establecidas en dos fragmentos de bosque seco (Tabla 1). Para el primer sitio se encontró registro de datos correspondientes al periodo 1995 al 2001; mientras otras parcelas establecidas en 2006 en otro fragmento generaron información adicional sobre cambio en las categorías de regeneración natural.

Tabla 1. Parámetros poblacionales y estructurales; y aspectos socioeconómicos relacionados al monitoreo de la especie Calycophyllum candidissimum

\begin{tabular}{lll}
\hline Poblacional & Estructural & Socioeconómico \\
\hline Mortalidad & Altura & Tipo de uso \\
Reclutamiento & Diámetro & Frecuencia de uso \\
Densidad & Categoría de regeneración \\
Incremento & Composición de la regeneración
\end{tabular}

Criterios e indicadores útiles para la ejecución y evaluación del monitoreo. De la información recopilada y analizada en los estudios que constituyen la línea base se proponen aspectos biológicos y de uso que por ser coincidentes en los trabajos revisados pueden constituirse en la base de futuros programas de monitoreo para especies con diferentes grados de amenaza.
Entre un amplio conjunto de factores eco fisiológicos relacionados a la presencia-ausencia, reproducción y productividad de C. candidissimum en su hábitat natural; se seleccionaron tres criterios (Tabla 1) y 12 indicadores útiles para la ejecución y evaluación para su monitoreo:

Dinámica poblacional. Relacionado al monitoreo del cambio estructural de una población en el tiempo, y asociado a elementos bióticos y abióticos del hábitat.

Gradiente ambiental. Referido a la determinación de la influencia de factores abióticos del medio que ayudan a identificar los factores limitantes de la distribución y abundancia de la especie.

Aspectos fenológicos. Cuya importanciaestá dirigida hacia la determinación de la eficiencia de estrategias reproductivas correlacionadas a la ocurrencia de eventos del clima en diferentes épocas del año y que determinan una cronosecuencia en la fenología de una especie.

Tabla 2. Criterios e indicadores útiles para ejecución y evaluación del monitoreo de Calycophyllum candidissimum

\begin{tabular}{|c|c|c|}
\hline Criterios & Indicadores & Parámetros para evaluación de indicadores \\
\hline Dinámica poblacional & $\begin{array}{l}\text { a) Densidad } \\
\text { b) Mortalidad } \\
\text { c) Reclutamiento } \\
\text { d) Incremento en biomasa } \\
\text { para el indicador incremento) } \\
\text { e) Intensidad de uso }\end{array}$ & $\begin{array}{l}\text { Reducción, Incremento (aplican para los tres } \\
\text { primeros indicadores) } \\
\text { Valores de IPA, IMA (definido para el } \\
\text { Baja, moderado, alto (definido para el } \\
\text { indicador intensidad de uso) }\end{array}$ \\
\hline $\begin{array}{l}\text { Gradiente ambiental } \\
\text { (relativo a la regeneración) } \\
\text { Natural }\end{array}$ & $\begin{array}{l}\text { a) Intensidad de luz } \\
\text { b) Topografía del sitio }\end{array}$ & $\begin{array}{l}\text { Totalmente expuesta Parcialmente } \\
\text { expuesta }(20-50 \%), \text { Sin luz }(\geq 50) \\
\text { Plano }(0-6 \%), \text { Moderadamente inclinado } \\
(6-25 \%), \text { Inclinada }(\geq 25 \%)\end{array}$ \\
\hline Aspectos fenológicos & $\begin{array}{l}\text { a) Temperatura } \\
\text { b) Porcentaje de Precipitación } \\
\text { c) Intensidad de luz } \\
\text { d) Viento } \\
\text { e) Brote y caída de } \\
\text { flores y frutos }\end{array}$ & $\begin{array}{l}\text { Baja, media, alta (temperatura) } \\
\text { Cantidad de lluvia caída en un mes con } \\
\text { respecto al total caído durante } 12 \text { meses } \\
\text { Grado de exposición (igual al gradiente } 2 \text { ) } \\
\text { Incidencia baja, media y alta (viento) } \\
\text { Presencia-ausencia del fenómeno }(0-100 \%)\end{array}$ \\
\hline
\end{tabular}




\section{RESULTADOS Y DISCUSIÓN}

Valores de abundancia de Calycophyllum candidissimum en diferentes tipos de vegetacion. A partir de las caracterizaciones realizadas en los estudios que constituyeron la línea base sobre la abundancia; la especie Calycophyllum candidissimum presenta una tendencia a encontrarse en bajas densidades (Tabla 3), y al igual que muchas especies, su densidad depende de la existencia de vegetación acompañante y principalmente del estado en que se encuentra el hábitat, en relación al uso actual del suelo. De manera general, la especie fue encontrada en los estados de desarrollo regeneración natural y fustal

Tabla 3. Información de línea base sobre abundancia de Calycophyllum candidissimum en paisajes boscosos fragmentados de Nicaragua

Componentes de paisajes boscosos fragmentados en Nicaragua

\begin{tabular}{cccccc}
\hline Sitio & $\begin{array}{c}\text { Bosque } \\
\text { secundario }\end{array}$ & $\begin{array}{l}\text { Bosque } \\
\text { ripario }\end{array}$ & $\begin{array}{c}\text { Áreas de } \\
\text { barbecho }\end{array}$ & $\begin{array}{c}\text { Potrero con } \\
\text { alta cobertura } \\
\text { arbórea }\end{array}$ & $\begin{array}{c}\text { Potrero con } \\
\text { baja cobertura } \\
\text { arbórea }\end{array}$ \\
\hline
\end{tabular}

Belén-Rivas.

\begin{tabular}{llllll} 
Sánchez et al., 2004 & 31 & 37 & 18 & 2 & 3 \\
\hline Matiguas-Matagalpa. & 38 & 9 & 7 & 4 & NR
\end{tabular}

Sánchez et al., 2005

Chacocente-Carazo.

Gonzalez-Rivas (2005)

0.56

NR

NR

NR

NR

Nandarola-Granada.

Noguera et al., 2009

$1.07^{\mathrm{f}}$

$3^{\mathrm{a}}$

NR

$0^{\mathrm{b}}$

NR

NR

${ }^{a}$ Abundancia en tres parcelas de 0.25 ha en una barbecho de 16 años

${ }^{\mathrm{b}}$ Abundancia en tres parcelas de 0.25 ha en un barbecho de 11 años

${ }^{\mathrm{d}}$ Abundancia relativa

${ }^{\mathrm{f}}$ Frecuencia relativa

NR No se registró en las parcelas inventariadas.

En un estudio realizado por Noguera et al., (2002) en dos áreas de bosque de galería en el departamento de Carazo la especie fue reportada con baja densidad; y de manera particular en una de las áreas con mayor influencia humana, la especie fue encontrada solamente en el estado de desarrollo latizal. Otro elemento de interés en relación a las diferencias en la abundancia es el patrón de distribución entre las distintas categorías de vegetacion para esta especie, que en su fase de regeneración presenta patrón de distribución agrupado, el que va cambiando a regular o aleatorio en la categoría fustal, lo que a la vez determina el número de árboles por unidad de área.

Cambio en la densidad de la especie a través del tiempo. La determinación del cambio en la densidad de individuos de la especie Calycophyllum candidissimum indica una ligera disminución de la población para un periodo de cinco años. En este sentido el resultado presentado corresponde a la disminución producto de mortalidad natural; sin embargo es importante considerar dentro de los factores que provocan disminución paulatina de poblaciones vegetales la intensidad en el uso, y el cambio que por la alteración continua del medio ambiente del bosque provoca alteraciones en los ciclos reproductivos de algunas especies.

Los valores de los parámetros poblacionales de la especie Calycophyllum candidissimum en el bosque seco secundario para un periodo de cinco años son los siguientes: mortalidad, 1.85 , reclutamiento, 0 , perdidas, 1.84, ganancias, 2.76 e incremento 2.76. Los resultados indican una tendencia a perdida en la densidad de individuos de la especie y baja recuperación de la misma debido a la inexistencia de reclutamiento como proceso de incremento o recuperación en la densidad de la población.

Factores de mortalidad e influencia sobre el área basal de la especie. Los principales factores de mortalidad identificados en arboles maduros se relacionan a infestación por lianas, y danos mecánicos provocados por el viento; mientras en las categorías de regeneración natural factores limitantes como baja humedad en el suelo y herbívoria son los más comunes. 
La pérdida en área basal se relaciona directamente con la mortalidad de la especie, mientras la ganancia e incremento en área basal es producto del incremento interno experimentado por los individuos de las diferentes categorías diametricas. Sin embargo, al relacionar este valor $(2.76 \%)$ con el incremento por categoría diametrica se puede afirmar que este es relativamente bajo.

Incremento de Calycophyllum candidissimum. Los valores de incremento periódico (IP) e incremento medio anual (IMA) muestran que para el periodo de estudio la especie presentó un mejor comportamiento en comparación a otras especies de valor ecológico dentro su hábitat natural.

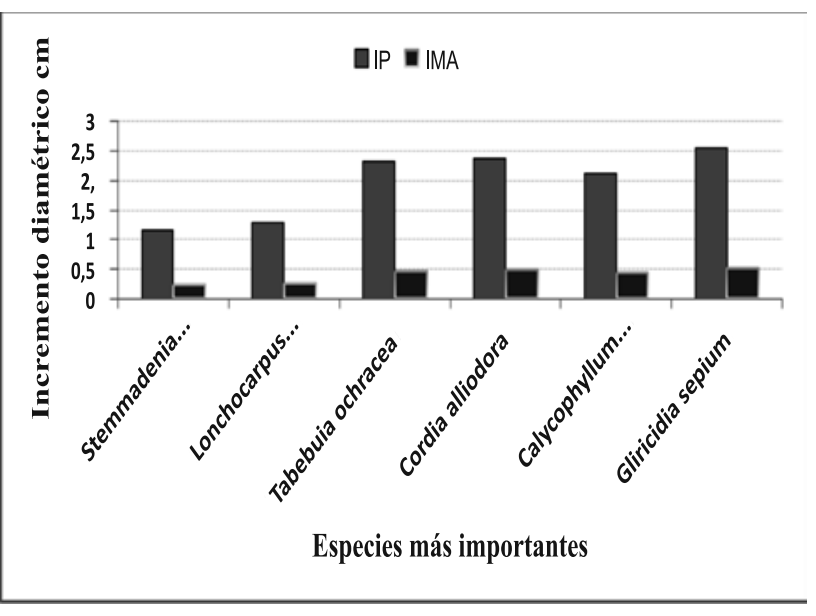

Figura 1. Valores del incremento periódico anual e incremento medio anual de cinco especies arbóreas del bosque seco secundario para un período de seis años.

Cambio en la regeneración natural a partir de factores ambientales luz y pendiente. La densidad de la población de brinzales y latizales experimentó un cambio positivo del 2001 al 2003, por lo que el valor de r para brinzales fue de 9 y para latizales fue de 5 (González-Rivas, 2008). La densidad media de la población de los brinzales y latizales varió significativamente en relación a la exposición de luz solar $\left(\mathrm{X}_{(0.05,2)}^{2}=48.71, \mathrm{p}<0.0001\right)$. La densidad de brinzales fue mucho mayor bajo la condición sin luz. En este sentido, fue posible observar que la especie prefiere sitios con baja intensidad de luz, lo cual reduce el impacto de altas temperaturas, por lo que se asume que la especie es menos vulnerables a la sequía (Gonzalez-Rivas, 2008).

En cuanto a la densidad media de la población en relación a la pendiente del terreno, se determinó que esta vario significativamente $\left(\mathrm{X}_{(0.05,2)}^{2}=27.56\right.$, $\mathrm{p}<0.0001)$. Las mayores densidades de brinzales y latizales fueron encontradas en áreas planas. Según González-Rivas, (2008) el tamaño de la semilla puede ser un factor a considerar para explicar las mayores densidades en sitios planos; ya que la semilla es muy pequeña y producto de la incidencia de factores como el viento y la escorrentía se prevé el movimiento de las semillas hacia estos sitios.

Con base en los datos sobre la regeneración de la especie, es posible concluir que la regeneración natural no es suficiente para restaurar densidades de la especie. Por lo que de acuerdo con FAO, CSFD, IPGRI, 2001 se debe implementar medidas como siembra directa, plantaciones de enriquecimientoyproteccióndelbosquedeincendiosypastoreo.

Plan de conservación y descripción de estrategias y actividades derivadas del monitoreo. Se presenta a continuaciónunapropuestadeplandeconservacióndelaespecie Calycophyllum candidissimum. Las estrategias y actividades incluidas tienen su fundamento en las actividades de monitoreo y de línea base descritas inicialmente por lo conllevan a una efectiva conservación y recuperación de las poblaciones.

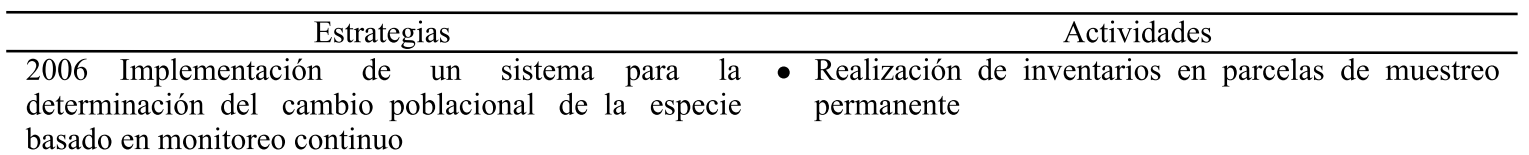

2010-2012 Protección de los fragmentos de bosque seco secundario

012-2013 Restablecimiento de poblaciones de la especie en fragmento de bosque seco
- Implementación de campanas de educación ambiental, dirigidas al conocimiento de la importancia del bosque seco como fuente de bienes y servicios

- Formación de brigadas contraincendios

- Implementación de prácticas de uso racional del recurso, a partir de la elaboración de planes de manejo de los fragmetos de bosque seco secundario

- Regular el uso de la especie a nivel local

- Recolección de semillas en sitios aledaños como forma de conservar y asegurar la variabilidad genética de la especie

- Construcción de viveros a nivel local

- Reintroducción de la especie en fragmentos de bosque seco secundario 
La presente propuesta considera además el aseguramiento o sostenibilidad de las estrategias, ya que dentro de las actividades se toman en cuenta la participación de las comunidades y además la obtención de beneficios que viabilicen los costos de implementación de las mismas.
Definición de buenas prácticas de manejo y conservación de la especie Calycophyllum candidissimum. La definición de buenas prácticas de manejo corresponde al espectro de aplicación de objetivos de conservación; permitiendo así formular micro proyectos y a la vez evaluar el grado de efectividad y eficiencia del plan de conservación.

Objetivo general: Asegurar la protección de los fragmentos de bosque seco secundario como estrategia de conservación de la especie Calycophyllum candidissimum

\section{Objetivos específicos}

Objetivo 1: Garantizar la conservación in situ de la especie Calycophyllum candidissimum así como el hábitat en el que se encuentra

Objetivo 2: Promover la implementación de medidas de manejo forestal del bosque seco secundario

Objetivo 3: Regular el uso y aprovechamiento de la especie a nivel local

\section{Actividades por objetivo}

Actividad 1: Realizar plantaciones de enriquecimiento en parches de bosque donde la especie esté ausente o presente baja densidad.

Actividad 2: Involucrar a la población en actividades de recolección de semillas de la especie y en la construcción de viveros como forma de conservación de las características genéticas de la especie, en cuanto a la adaptación a las condiciones locales.

Actividad 3: Implementar actividades de prevención de incendios forestales y control de pastoreo en fragmentos de bosque seco secundario para proteger la regeneración natural.

Actividad 1: Integrar el manejo silvicultural del bosque seco secundario a la dinámica de producción o generación de bienes de las fincas.

Actividad 2: Promover que el plan general de manejo se convierta en un verdadero instrumento de conservación de especies de alto valor comercial.

Actividad 1: Regular las actividades humanas con carácter comercial en relación al potencial de afectación al bosque: pastoreo, obtención de leña, medicinas, entre otras.

Actividad 2: Promover la aplicación de la normativa técnica obligatoria en cuanto a concesión local de permisos para cambio de uso del suelo. 


\section{REFERENCIAS BIBLIOGRÁFICAS}

Castro-Marín, G; Nygard, R; Gonzáles Rivas, B; Oden- Chister, P. 2005. Stand dynamics and basal areachange in a tropical dryforest Reserve in Nicaragua. ForestEcology and Management, 208. 63-75.

FAO (Organizacion de las Naciones Unidas para la Agricultura y la Alimentación), CSFD (Centro de semillas forestales de Danida), IPGRI (Instituto internacional de recursos fitogenéticos). 2001. Conservación y ordenación de recursos genéticos forestales: en bosques naturales ordenados y áreas protegidas (in situ). Instituto Internacional de recursos fitogenéticos. Inglaterra. $94 \mathrm{pp}$.

González-Rivas, B. 2005. Treediversity and regeneration in tropical dryforest of Nicaragua. Doctoral Thesis. Umae. Pág. 1-10

Gonzalez-Rivas, B. 2008. Regeneración natural del Madroño (Calycophyllumcandidissimum) (Vahl DC) en el bosque tropical seco de Chacocente, Nicaragua. La Calera. Ano 8. No. 9

Noguera, A; Castro, G; González, B. 2002. Diversidad Florística del Bosque de Galería en Dos Localidades del Departamento de Carazo, Nicaragua. LA CALERA. Revista científica de la Universidad Nacional Agraria 4(4).

Noguera-Talavera, A, Obando, R, Oliva, E. 2009. Composición, estructura y dinámica de dos sitios de bosque seco secundario en el Suroeste de Nicaragua: Pautas para el diseño e implementación de alternativas de manejo. LA CALERA. 9:12. Pág. 5-11.

Ponce, VL. Montalbán, H. 2005. Banco de semillas del suelo de tres sitios de Nandarola, Nandaime. Trabajo de Diploma. UNA-FARENA. Managua, Nicaragua. 23 Pág.

Sánchez, D, Harvey, C; Grijalva, A; Medina, A; Vílchez, S; Hernández, B. 2005. Diversidad, composición y estructura de la vegetación en un agropaisaje ganadero en Matiguas, Nicaragua. Biología Tropical. Vol. 53:3. Pág. 387-414.

Sánchez, D. Harvey, C. Grijalva, A. Medina, A. Vílchez, S. Hernández, B. 2004. Diversidad, composición y estructura de la vegetación en un paisaje. 